\title{
Combined Effects of Gastrocnemius Stretch and Tibialis Anterior Resistance Exercise in Subjects with Limited Ankle Dorsiflexion
}

\author{
Jihyun Lee ${ }^{a}$, Heonseock Cynn ${ }^{b}$, Areum Shin ${ }^{b}$, Bobeen Kim ${ }^{b}$ \\ aDepartment of Physical Therapy, Baekseok University, Baekseokdaehak-ro, Dongnam-gu, Cheonan-si, Chungcheongnam-do, Republic \\ of Korea \\ ${ }^{\mathbf{b}}$ Applied Kinesiology and Ergonomic Technology Laboratory, Department of Physical Therapy, Graduate School, Yonsei University, \\ Won-ju, Republic of South Korea.
}

\begin{abstract}
Objective: Limited ankle dorsiflexion is related to ankle injuries. There are various exercises to increase the flexibility of the gastrocnemius for improving the passive range of motion in ankle dorsiflexion. However, to performances in daily activities and athletic sports and higher efficiency of walking and running, both ankle dorsiflexion passive and active range of motion are needed. To investigate the effects of combined gastrocnemius stretching and tibialis anterior resistance exercise on ankle kinematics (passive and active range of motion of ankle dorsiflexion) and tibialis anterior muscle activity in subjects with limited ankle dorsiflexion.
\end{abstract}

Design: Cross-sectional single-group repeated measures design.

Methods: Fourteen subjects with limited ankle dorsiflexion were recruited (in the right ankle in 7 and the left ankle in 7). All subjects performed gastrocnemius stretching alone and tibialis anterior resistance exercise after gastrocnemius stretching. The passive and active range of motion of ankle dorsiflexion were measured after interventions immediately. The tibialis anterior activity was measured during active range of motion of ankle dorsiflexion measurement.

Results: There was no significant difference of ankle dorsiflexion passive range of motion between gastrocnemius stretching alone and the tibialis anterior resistance exercise after gastrocnemius stretching. The tibialis anterior resistance exercise after gastrocnemius stretching significantly increased active range of motion of ankle dorsiflexion compared to gastrocnemius stretching alone $(\mathrm{p}<0.05)$. The tibialis anterior resistance exercise after gastrocnemius stretching significantly increased tibialis anterior activity better than did gastrocnemius stretching alone.

Conclusions: Thus, subjects with limited ankle dorsiflexion should be encouraged to perform tibialis anterior resistance exercises.

Key Words: Ankle, Muscle Stretching Exercises, Resistance.

\section{Introduction}

Limited ankle dorsiflexion (DF) is related to ankle injuries such as ankle sprain, Achilles tendinitis, and plantar fasciitis [1-3]. The risk factors of limited ankle DF were reported to be tightness of the calf muscle, loss of mobility in the soft tissue or capsular ligament, and loss of talar posterior glide [4, 5]. Many previous studies have examined the effectiveness of various exercises for increasing the flexibility of the gastrocnemius (GCM) to improve the range of motion (ROM) in ankle DF, including stretching the GCM and joint mobilization [6-9]. Previous researchers recommended GCM stretching increase ankle DF passive ROM

Received: Feb 2, 2021 Revised: Feb 24, 2021 Accepted: Mar 4, 2021

Corresponding author: Heonseock Cynn (ORCID https://orcid.org/0000-0002-5810-2371)

Applied Kinesiology and Ergonomic Technology Laboratory, Department of Physical Therapy, Graduate School, Yonsei University. Wonju, Gangwon-do 220-710, Republic of South Korea.

TEL: +82-33-760-2427 Fax: +82-33-760-2496 E-mail: cynn@yonsei.ac.kr

This is an Open-Access article distributed under the terms of the Creative Commons Attribution Non-Commercial License (http://creativecommons.org/licenses/ by-nc/4.0) which permits unrestricted non-commercial use, distribution, and reproduction in any medium, provided the original work is properly cited.

Copyright @ 2021 Korean Academy of Physical Therapy Rehabilitation Science 
(DF PROM) during the GCM stretch.

However, to improve performances in daily activities and sports, and increase the efficiency of walking and running, both DF PROM and active ROM (AROM) are needed [10-12]. In the presence of a short GCM, tibialis anterior (TA) activity could be inhibited when the ankle is dorsiflexed. TA resistance exercise might be useful to increase TA muscle activity and ankle DF PROM/ AROM in subjects with limited ankle DF. Even as this technique might be helpful, no previous studies have investigated a strategy to increase the flexibility of the GCM and the activation of the TA in subjects with limited ankle DF.

The purpose of this study was to compare the effects of GCM stretching alone and GCM stretching with TA resistance exercise (GCM stretching + TA) on the ankle kinematics (PROM/AROM) and TA muscle activity in subjects with limited ankle DF. We hypothesized that ankle PROM/AROM and TA muscle activity would increase after GCM stretching + TA more than with GCM stretching alone in subjects with limited ankle DF.

\section{Methods}

\section{Participants}

In total, 14 subjects with limited ankle DF (in the right ankle in 7 and the left ankle in 7) participated. For this study, 41 university student volunteers were recruited through advertisement on the group social media in the university, and examiners measured ankle DF PROM in all volunteers. Participants with $<10^{\circ}$ of ankle DF PROM with the knee extended in the prone position were eligible for this study [13, 14]. Among 41 volunteers, 21 volunteers were people with no limited ankle DF. The six volunteers reported a history of ankle sprain within the past 6 months and were excluded, leaving a total of 14 participants. Among the 14 participants, 5 participants had bilateral limited ankle DF PROM. We decided that an ankle had the worse DF PROM when a bilateral comparison indicated a difference in DF PROM of $<1^{\circ}$. Exclusion criteria were lower extremity surgical history, fractures, neurological diseases, hip and/or knee flexion contracture, or musculoskeletal soft tissue injury of the lower extremity, such as ankle sprain within the previous 6 months. Those who complained of discomfort or disagree with this study procedure were excluded from the study. The G-power software (G-power ver. 3.1.6; Heinrich-HeineUniversität Düsseldorf, Germany) was used to calculate the sample size. By using data obtained from a pilot study of five participants, we calculated the necessary sample size to be 10 participants to achieve a power of 0.80 and an effect size of 0.85 (calculated from the partial 2 of 0.42 from the pilot study), with an $\alpha$ level of 0.05 . Prior to participation, the participants provided written informed consent and the principal investigator explained the experimental procedure and safety to all participants. All participants signed an informed consent form approved by Yonsei University Institutional Review Board (1041849-201708-BM-088-02).

\section{Procedures}

This study was a cross-sectional single-group repeated measures design. Ankle DF PROM was measured by using a 14-in stainless steel goniometer. The subjects were positioned prone on the treatment table, with the foot extending beyond the end of the table. The investigator maintained a neutral subtalar joint position and applied force to the plantar surface of the forefoot and midfoot until further movement was firmly resisted. The fulcrum of the goniometer was placed over the lateral malleolus, and the stationary and moving arms were aligned with the fibular head and parallel to the fifth metatarsal, respectively. The three points were marked with dots by using a pen. The three marked dots were retained until the end of the study [15]. For measurement of ankle DF AROM, the subject was asked in a long sitting position and knees fully extended, a bolster was placed at about popliteal fossa and heel level to allow full ROM for DF and plantar flexion. The principal investigator confirmed a neutral subtalar joint position. Then, the investigator instructed the subjects to perform active DF as needed to allow for maximal ankle DF in the test. A second investigator confirmed the ankle DF AROM measurement. All measurements were performed twice; the goniometer was removed after each measurement. The mean value of two ankle DF ROM measurements was used for data analysis (reliability intraclass correlation coefficient $=0.95$ ) [7, 14].

Surface electromyography (EMG) data were collected by using a Noraxon TeleMyo-DTS (TeleMyo-DTS; 
Noraxon Inc., USA) and analyzed by using the Noraxon MyoResearch 1.06 XP software. The EMG signals were amplified, band-pass filtered (10 and $450 \mathrm{~Hz}$ ), and notch filtered ( 60 and $120 \mathrm{~Hz}$ ) before digitally recorded at 1000 $\mathrm{Hz}$ and processed into root mean square values. The TA electrodes were placed at one-third the distance between the head of the fibula and the tip of the medial malleolus on the measured leg. Two electrodes were placed approximately $20 \mathrm{~mm}$ apart in the direction of the muscle fibers. Data on maximal voluntary isometric contraction (MVIC) were collected to normalize the EMG data on the TA by using the manual muscle testing positions. To obtain the MVIC value for TA, the investigator held the subject's distal calf with one hand and, with the other hand, applied resistance on the medial side and dorsal surface of the subject's foot in the direction of plantar flexion of the ankle joint and eversion of the foot in a sitting position. Each contraction was held for 5 seconds, with maximal effort against manual resistance, and a 2-minute rest was given between trials to minimize muscle fatigue. The mean MVIC value in the two trials was calculated. The first and last second of EMG data from each MVIC trial were discarded, and the remaining 3 seconds of data were used. The collected EMG amplitudes from the TA muscles during the exercise were expressed as a percentage of the mean MVIC (\%MVIC). The ankle DF P/AROMs were measured after interventions immediately. The tibialis anterior activity was measured during ankle DF AROM measurement. The test-retest reliabilities for EMG measurement of TA muscle were substantial in two conditions (GCM stretching alone: GCM stretching + TA).

For GCM stretching alone, the subjects were instructed to stand on two scales. The amount of body weight on the tested leg (the side with limited ankle DF PROM with the knee extended) was maintained at $60 \%$

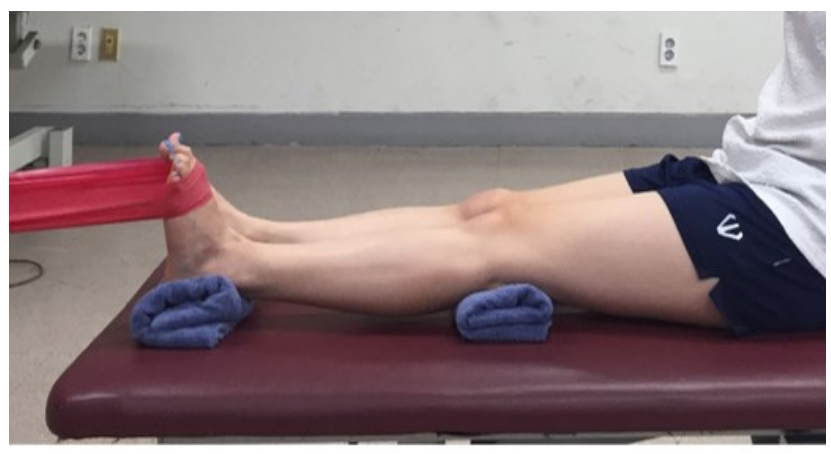

Figure 1. Passive joint mobilization $\pm 5 \%$ of the subject's body weight by using scales to generalize the force applied to the tested leg during stretching [15]. The tested leg was placed one step length behind the contralateral leg. If the right ankle was limited, the right leg was placed one step length backward to stretch. The subjects were asked to lean forward toward the wall with contralateral knee flexion until they felt a maximal tolerable stretch of the GCM in the tested leg [10]. The subjects placed their hands on the wall for balance and maintained a straight knee without heel-off in the tested leg during the stretching. Each stretch was held for 30 seconds in all five trials. The parameters of the stretching exercises were based on previous investigator data regarding hamstring stretching exercises. The subjects were allowed a 10 -second rest period between stretches [16]. For the TA resistance exercise after GCM stretching (GCM stretching + TA), the subjects performed the intervention in the same way as in the GCM stretching alone, except for the additional TA resistance exercise. For the TA resistance exercise, the subjects sat on the bed with one end of the band wrapped around the treatment table and the other end around the metatarsal heads of the involved foot. With the subject in a long sitting position and their knees fully extended, a towel was placed at about popliteal fossa and heel level to allow full ROM for DF and plantar flexion. Thera-band was stretched to an additional $70 \%$ of its resting length to allow for consistent resistance tension among the subjects. The subjects were instructed to use only the involved ankle joint and avoid toe extensions at the metatarsophalangeal joint for pure ankle DF by the TA muscle, as toe extension by the extensor hallucis longus (EHL) and extensor digitorum longus can inhibit TA activation. The subjects maintained a consistent pace of approximately 3 to 5 seconds per repetition throughout the full ROM [17] (Figure 1). They were then allowed to familiarize themselves with the exercises for approximately $10 \mathrm{~min}$. A 5-min rest period was allowed after the familiarization period and before data collection began.

Data analysis

The PASW Statistics 22 software (SPSS Statistics 22.0; SPSS inc., USA) was used in all the statistical analyses. Normality test was performed using the Kolmogorov-Smirnov test. The data were normally 
Table 1. Changes in passive and active range of motion of ankle dorsiflexion and tibialis anterior muscle activity between gastrocnemius stretching alone and the tibialis anterior resistance exercise after gastrocnemius stretching $(n=14)$

\begin{tabular}{lllll}
\hline Parameter & GCM stretching alone & GCM stretching + TA & changes & t (p) \\
\hline PROM $\left(^{\circ}\right)$ & $-23.71(5.05)$ & $-24.29(4.32)$ & 0.57 & $0.401(0.698)$ \\
AROM $\left(^{\circ}\right)$ & $-16.29(6.67)$ & $-13.21(5.75)$ & 3.08 & $2.199(0.047)$ \\
TA $(\% M V I C)$ & $39.12(21.73)$ & $51.31(15.32)$ & 12.19 & $-2.748(0.017)$ \\
\hline
\end{tabular}

Values are presented as mean (SD).

A positive angles indicates dorsiflexion, negative angles indicates plantarflexion because subjects had limited ankle dorsiflexion.

GCM stretching alone: gastrocnemius stretching alone, GCM stretching + TA: tibialis anterior resistance exercise after gastrocnemius stretching, PROM: passive range of motion of ankle dorsiflexion, AROM: active range of motion of ankle dorsiflexion, TA: tibialis anterior.

$\mathrm{p}<0.05$.

distributed, and the parameter test was performed. The paired t-test was used to compare dependent variables. The level of significance was set at 0.05 .

\section{Results}

There was no significant difference of ankle DF PROM between GCM stretching alone and GCM stretching + TA. The GCM stretching + TA significantly increased ankle DF AROM and TA activity better than did GCM stretching alone $(\mathrm{p}<0.05)($ Table 1$)$.

\section{Discussion}

Primary finding of this study was that GCM stretching + TA significantly increased ankle DF AROM and TA activity better than did GCM stretching alone.

We found that GCM stretching + TA significantly increased ankle DF AROM better than did GCM stretching alone (18.91\%). This finding supports the original research hypothesis. The previous study reported that DF AROM significantly increased in static calf muscle stretching and dorsiflexor resistance training program [18]. Ankle DF AROM requires action of the dorsiflexor muscles and the flexibility of the calf muscles. TA muscle activity increased after including TA resistance exercise. This was confirmed by the surface EMG measurement of TA muscle. Another explanation was autogenic facilitation of TA followed by reciprocal inhibition of GCM during GCM stretching. Through reciprocal innervation, the opposite muscles of the GCM, that is, the TA, was reciprocally facilitated. This study used active application of metatarsophalangeal joint flexion during DF to avoid activation of EHL and extensor digitorum longus. Thus, metatarsophalangeal joint flexion could facilitate TA activation. Owing to the increased TA muscle activity, the ankle DF AROM increased. Taking into account the results, TA resistance exercise should be included to increase ankle DF AROM and GCM stretching in subjects with limited ankle DF.

TA activity significantly increased with GCM stretching + TA better than with GCM stretching alone by $31.16 \%$. This finding also supports the original research hypothesis. A previous study demonstrated increased isometric maximal voluntary dorsiflexion torque after static calf stretching and resistance training program for 6 weeks, although muscle activity was not measured [19]. We propose several possible explanations for the increased activity of the TA shortly after including TA resistance exercise. First, the shortness of the GCM is known to limit ankle DF. Thus, after including TA resistance exercise, ankle DF AROM was increased. These were confirmed above this session. Owing to the increased range, the TA muscle continued to contract. Second, the neurophysiological concept of reciprocal innervation $[20,21]$ can likely explain the increase in TA activity. When GCM stretching was maintained for 30 seconds in this study, the Golgi tendon organ might have been activated and induced autogenic inhibition of the GCM. Through reciprocal innervation, the opposite muscles of the GCM, that is, the TA, was reciprocally facilitated. Hence, this study suggests that combination of GCM stretching and TA resistance exercise is an effective method for facilitating 
TA activity in subjects with limited ankle DF.

This study has several limitations. First, owing to its cross-sectional design, this study could not determine the long-term effects of GCM stretching with TA resistance exercise of the findings of the present study. Thus, future studies should be conducted to investigate the long-term effects of GCM stretching alone and GCM stretching + TA on ankle kinematics and TA muscle activity. Second, this study did not measure TA weakness before and after the interventions, although limited DF could influence TA weakness or vice versa. Thus, future study should consider TA weakness measurement.

In conclusion, the results indicate that GCM stretching + TA could be more effective methods than GCM stretching alone for improving ankle DF AROM and TA activity in subjects with limited ankle DF. Thus, patients with limited ankle DF should be encouraged to perform TA resistance exercise after GCM stretching.

\section{References}

1. Kibler WB, Goldberg C, Chandler TJ. Functional biomechanical deficits in running athletes with plantar fasciitis. Am J Sports Med. 1991;19(1):66-71.

2. Schepsis AA, Jones H, Haas AL. Achilles tendon disorders in athletes. Am J Sports Med. 2002;30(2): 287-305.

3. Willems TM, Witvrouw E, Delbaere K, Mahieu N, De Bourdeaudhuij I, De Clercq D. Intrinsic risk factors for inversion ankle sprains in male subjects a prospective study. Am J Sports Med. 2005;33(3):415-23.

4. Sahrmann S. Movement system impairment syndromes of the extremities, cervical, and thoracic spines. St. Louis, Mo.: Elsevier/Mosby; 2011.

5. Denegar CR, Hertel J, Fonseca J. The effect of lateral ankle sprain on dorsiflexion range of motion, posterior talar glide, and joint laxity. J Orthop Sports Phys Ther. 2002;32(4):166-73.

6. Harkey M, McLeod M, Van Scoit A, Terada M, Tevald M, Gribble P, et al. The immediate effects of an anterior-to-posterior talar mobilization on neural excitability, dorsiflexion range of motion, and dynamic balance in patients with chronic ankle instability. J sport Rehabil. 2014;23(4):351-59.
7. Kang MH, Kim JW, Choung SD, Park KN, Kwon OY, Oh JS. Immediate effect of walking with talus-stabilizing taping on ankle kinematics in subjects with limited ankle dorsiflexion. Phys Ther Sport. 2014;15:156-61.

8. Marrón-Gómez D, Rodríguez-Fernández ÁL, MartínUrrialde JA. The effect of two mobilization techniques on dorsiflexion in people with chronic ankle instability. Phys Ther Sport. 2015;16(1):10-5.

9. Weppler $\mathrm{CH}$, Magnusson SP. Increasing muscle extensibility: a matter of increasing length or modifying sensation? Phys Ther. 2010;90(3):438-49.

10. Gajdosik RL, Allred JD, Gabbert HL, Sonsteng BA. A stretching program increases the dynamic passive length and passive resistive properties of the calf muscle-tendon unit of unconditioned younger women. Eur J Appl Physiol. 2007;99(4):449-54.

11. Rodacki AL, Souza RM, Ugrinowitsch C, Cristopoliski F, Fowler NE. Transient effects of stretching exercises on gait parameters of elderly women. Man Ther. 2009;14(2):167-72.

12. Tainaka K, Takizawa T, Katamoto S, Aoki J. Six year prospective study of physical fitness and incidence of disability among community-dwelling Japanese elderly women. Geriatr Gerontol Int. 2009;9(1):21-8.

13. Dinh NV, Freeman H, Granger J, Wong S, Johanson M. Calf stretching in non-weight bearing versus weight bearing. Int J Sports Med. 2011;32:205-10.

14. Kang MH, Oh JS, Kwon OY, Weon JH, An DH, Yoo WG. Immediate combined effect of gastrocnemius stretching and sustained talocrural joint mobilization in individuals with limited ankle dorsiflexion: A randomized controlled trial. Man Ther. 2015;20:827-34.

15. Jung DY, Koh EK, Kwon OY, Yi CH, Oh JS, Weon JH. Effect of medial arch support on displacement of the myotendinous junction of the gastrocnemius during standing wall stretching. J Orthop Sports Phys Ther. 2009;39:867-74.

16. Bandy WD, Irion JM, Briggler M. The effect of time and frequency of static stretching on flexibility of the hamstring muscles. Phys Ther. 1997;77(10):1090-6.

17. Hall EA, Docherty CL, Simon J, Kingma JJ, 
Klossner JC. Strength-training protocols to improve deficits in participants with chronic ankle instability: a randomized controlled trial. J Athl Train. 2015; 50(1):36-44.

18. Youdas JW, Krause DA, Egan KS, Therneau TM, Laskowski ER. The effect of static stretching of the calf muscle-tendon unit on active ankle dorsiflexion range of motion. J Orthop Sports Phys Ther. 2003;33(7):408-17.

19. Kato E, Kurihara T, Kanehisa H, Fukunaga T, Kawakami Y. Combined effects of stretching and resistance training on ankle joint flexibility. Physiology Journal. 2013;

20. Bell J. The anatomy and physiology of the human body. Vol. 1. Collins \& Company, NY, USA.

21. Sherrington CS. Note on the knee-jerk and the correlation of action of antagonistic muscles. Proc. R. Soc. Lond. 1892;52(315-320):556-64. 